\title{
Development of Diagnostics for High-Temperature High-Pressure Liquid Pb-16Li Applications
}

\author{
Abhishek Saraswat ${ }^{1}$, Srikanta Sahu ${ }^{1}$, T. Srinivas Rao' ${ }^{1}$, Ashokkumar Prajapati1 ${ }^{1}$ Shrikant Verma ${ }^{1}$, \\ Sandeep Gupta1, Mritunjay Kumar1, Rajendraprasad Bhattacharyay', Partha Das ${ }^{2}$
}

${ }^{1}$ Institute for Plasma Research, Gandhinagar, India

${ }^{2}$ Bhabha Atomic Research Centre, Mumbai, India

Email: asaraswat@ipr.res.in

How to cite this paper: Saraswat, A., Sahu, S., Rao, T.S., Prajapati, A., Verma, S., Gupta, S., Kumar, M., Bhattacharyay, R. and Das, P. (2017) Development of Diagnostics for High-Temperature High-Pressure Liquid $\mathrm{Pb}-16 \mathrm{Li}$ Applications. World Journal of Nuclear Science and Technology, 7, 292-308. https://doi.org/10.4236/wjnst.2017.74023

Received: September 20, 2017

Accepted: October 17, 2017

Published: October 20, 2017

Copyright $\odot 2017$ by authors and Scientific Research Publishing Inc. This work is licensed under the Creative Commons Attribution International License (CC BY 4.0).

http://creativecommons.org/licenses/by/4.0/

\section{(c) (i) Open Access}

\begin{abstract}
Liquid lead-lithium $(\mathrm{Pb}-16 \mathrm{Li})$ is of primary interest as one of the candidate materials for tritium breeder, neutron multiplier and coolant fluid in liquid metal blanket concepts relevant to fusion power plants. For an effective and reliable operation of such high temperature liquid metal systems, monitoring and control of critical process parameters is essential. However, limited operational experience coupled with high temperature operating conditions and corrosive nature of $\mathrm{Pb}$-16Li severely limited application of commercially available diagnostic tools. This paper illustrates indigenous calibration test facility designs and experimental methods used to develop non-contact configuration level diagnostics using pulse radar level sensor, wetted configuration pressure diagnostics using diaphragm seal type pressure sensor and bulk temperature diagnostics with temperature profiling for high temperature, high pressure liquid $\mathrm{Pb}$ and $\mathrm{Pb}-16 \mathrm{Li}$ applications. Calibration check of these sensors was performed using analytical methods, at temperature between $380^{\circ} \mathrm{C}-400^{\circ} \mathrm{C}$ and pressure upto $1 \mathrm{MPa}(\mathrm{g})$. Reliability and performance validation were achieved through long duration testing of sensors in liquid $\mathrm{Pb}$ and liquid $\mathrm{Pb}-16 \mathrm{Li}$ environment for over 1000 hour. Estimated deviation for radar level sensor lies within $[-3.36 \mathrm{~mm},+13.64 \mathrm{~mm}]$ and the estimated error for pressure sensor lies within $1.1 \%$ of calibrated span over the entire test duration. Results obtained and critical observations from these tests are presented in this paper.
\end{abstract}

\section{Keywords}

Sensor, Lead-Lithium, Liquid Metal, Fusion Blanket 


\section{Introduction}

One of the key missions of International Thermonuclear Experimental Reactor (ITER) is to validate the design concepts for tritium breeding blankets relevant to a power producing reactor like DEMO. ITER operation will demonstrate feasibility of breeding blanket concepts that would lead to tritium self-sufficiency and high-grade heat extraction, which are necessary goals for DEMO [1] [2] [3]. India is working towards development of Lead Lithium Ceramic Breeder (LLCB) Test Blanket Module (TBM), planned to be tested in equatorial port \#2 in ITER [1]. LLCB blanket concept consists of lithium titanate ceramic breeder in the form of packed pebble bed with liquid $\mathrm{Pb}$-16Li alloy eutectic (hereafter referred to as $\mathrm{Pb}-\mathrm{Li}$ ) acting as a tritium breeder, neutron multiplier and coolant. To achieve the intended operation of LLCB blanket, $\mathrm{Pb}-\mathrm{Li}$ is circulated in a closed loop, called Lead Lithium Cooling System (LLCS), which extracts the volumetric heat generated within TBM internal structures along with its self-generated neutronic heat. LLCS is being designed to operate at a gauge pressure of $1.2 \mathrm{MPa}$ with temperatures between $300^{\circ} \mathrm{C}-460^{\circ} \mathrm{C}$ at TBM inlet and outlet, respectively, to extract a total heat load of $0.38 \mathrm{MW}$. Process parameters like pressure, level, temperature and flow need to be monitored and controlled for an effective functioning of LLCS as a primary cooling system. However, limited instrumentation availability for liquid metal applications and aggressive operating parameters of LLCS coupled with corrosive nature of $\mathrm{Pb}-\mathrm{Li}$ presents additional major challenges for process sensors and diagnostic tools. Additionally, typical interval of 2 years between scheduled maintenance cycles of ITER [4] demands precise validation and reliable performance of sensors over long duration operation. Heavy liquid metal coolants based on lead mandate the development of specially adapted and properly validated diagnostics. Although a number of validated measurement technologies are available for liquid sodium coolant, these technologies need to be further developed for application in liquid lead and lead-lithium coolants because of substantial difference in properties and required operating parameters [5]. As a first step to address some of these challenges, experimental studies were carried out towards calibration and rigorous performance validation of sensors as part of process instrumentation development for static liquid $\mathrm{Pb} / \mathrm{Pb}$ - Li applications. To fulfil the test objectives, calibration test facilities were designed and fabricated at Institute for Plasma Research (IPR). Calibration tests and long duration tests for over 1000 hour were carried out to assess the performance as well as operational reliabilities of pressure sensors, level sensor and temperature sensor assembly in liquid $\mathrm{Pb} / \mathrm{Pb}-\mathrm{Li}$ environments relevant to LLCS. Calibration test facility designs and selected sensor configurations are discussed in this paper. Process test parameters, test procedures and long duration performance data obtained for tested sensors are also presented.

\section{Sensor Selection}

Application of $\mathrm{Pb}-\mathrm{Li}$ is primarily confined to fusion specific studies [6]. In addi- 
tion, corrosive nature and high operational temperature requirements have largely impeded development of process instrumentation for Pb-Li. Proper selection of measurement technique, sizing of sensor, engineering modification/ customization of Commercial Off-The-Shelf (COTS) sensors as applicable for specific requirements, installation considerations demanded by liquid metal applications and rigorous experimental validation in $\mathrm{Pb}-\mathrm{Li}$ environment should be addressed to establish compatibility, to validate application feasibility and to efficaciously develop any potential measurement technology relevant to liquid $\mathrm{Pb}-\mathrm{Li}$ application.

\subsection{Pressure Measurement}

For a wetted pressure sensor in high temperature environment, the sensing element along with signal conditioning electronics must be isolated from extreme temperature [7]. To achieve this, an indirect liquid metal pressure measurement approach was developed using gas pressure measurements [8]. However, such an approach essentially requires tight control over temperature to minimize errors from temperature dependent gas pressurization in a fixed volume, which may be sometimes difficult to achieve during steady state operation. In the present study, we have adopted piezo-resistive principle based remote diaphragm seal type pressure sensors with remotely mounted electronics for pressure measurement of liquid $\mathrm{Pb} / \mathrm{Pb}$-Li. Wetted diaphragm, acting as a process isolation seal, transmits pressure upto remotely mounted sensing diaphragm of piezo-resistive sensor through a fine capillary filled with an incompressible, high temperature compatible fluid. Minimum volume displacement of capillary fill fluid ensures better dynamic response towards fast changes in pressure, which is satisfactory for LLCS operational point of view. In this study, two types of pressure sensors with 0 - $2 \mathrm{MPa}$ (g) calibrated range were selected: a customized remote diaphragm seal type pressure sensor with a 1-inch SS-316 wetted diaphragm with 0.25 $\mathrm{mm}$ bore rigid stem configuration capillary containing sodium-potassium alloy $(\mathrm{NaK})$ as shown in Figure 1(a) and remote diaphragm seal type pressure sensor with a 2 -inch SS-316L wetted diaphragm with $1 \mathrm{~mm}$ bore flexible capillary containing high temperature compatible silicone oil as shown in Figure 1(b). Both the sensors are equipped with current transmitters to provide a linear output signal in the calibrated range.

\subsection{Level Measurement}

A variety of validated level diagnostics like conductivity probes, induction sensors etc. are available for a few liquid metal applications like $\mathrm{Na} / \mathrm{NaK}$ etc. [7]. However, in $\mathrm{Pb}$-Li environment, a wetted configuration level diagnostics maybe rendered ineffective over long durations due to deposition of metallic oxides, corrosion of sensor probe and bending stresses exerted by high density liquid $\mathrm{Pb} / \mathrm{Pb}-\mathrm{Li}$.

Hence, to continuously measure level in a non-contact manner, a pulse radar level sensor equipped with a linear current transmitter was selected. It is based 


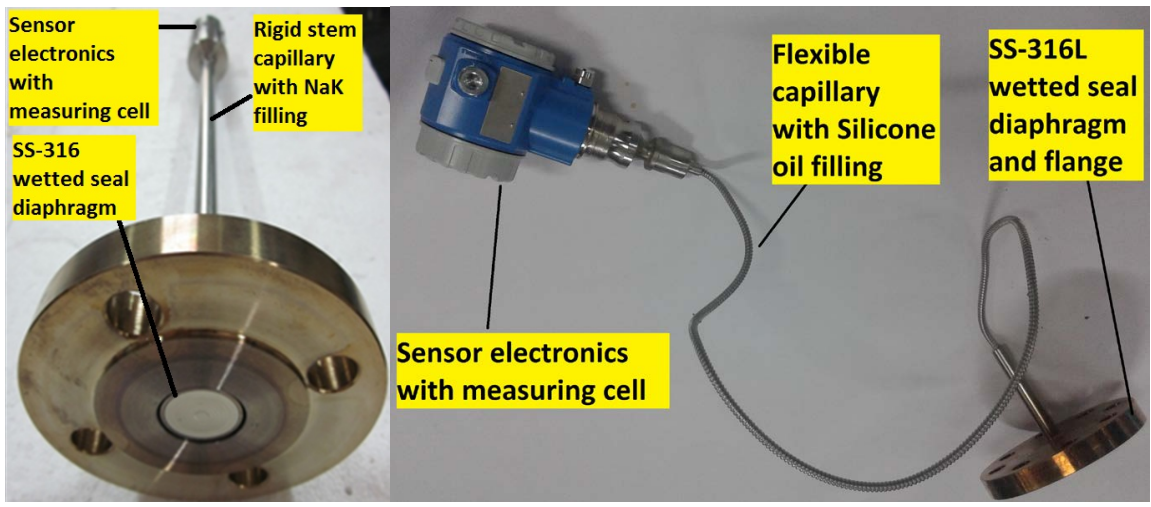

(a)

(b)

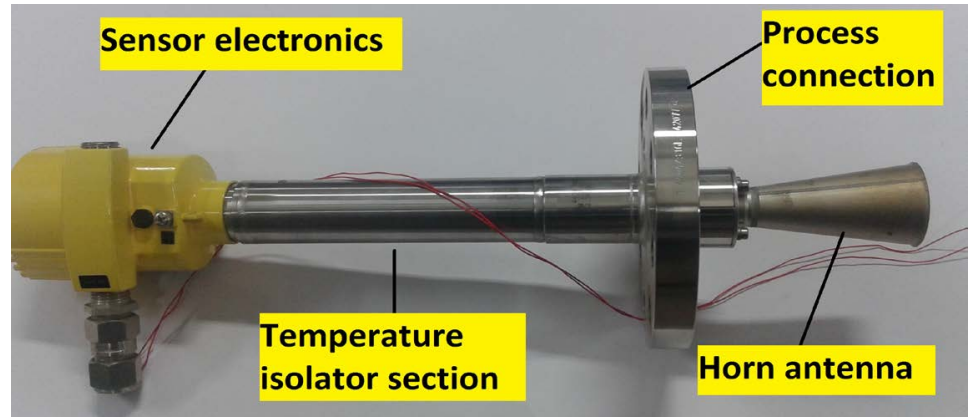

(c)
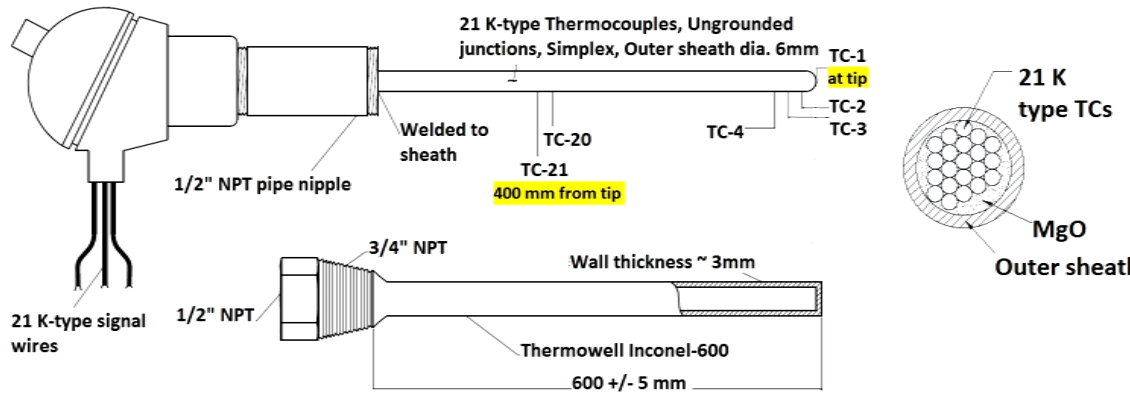

(d)

Figure 1. (a) NaK fill fluid based remote diaphragm seal type pressure sensor; (b) High temperature compatible silicone oil fill fluid based remote diaphragm seal type pressure sensor; (c) Non-contact type pulse radar level sensor with temperature isolator section to decouple high process temperatures; (d) TeLePro assembly with multiple temperature measuring junctions (K-type) for bulk temperature measurements and liquid metal level estimation.

on the principle of measuring transit time between the emitted microwave pulse and its reflected echo from the sensed material surface. The transit time is directly proportional to distance between sensor and the medium [9]. Unlike ultrasonic level sensors based on time of flight principle, radar level sensor provides a non-contact technique virtually unaffected by variations in process temperature, pressure or cover gas composition because of negligibly small variation in the dielectric constant of propagation medium as determined by Equation (1),

$$
\varepsilon_{r}=1+\left[\left(\varepsilon_{r N}-1\right) \cdot\left(\theta_{N} \cdot P\right) /\left(\theta \cdot P_{N}\right)\right]
$$


where, $\varepsilon_{r}$ is calculated dielectric constant, $\boldsymbol{\varepsilon}_{r N}$ is dielectric constant of gas/vapour under normal conditions (temperature $273 \mathrm{~K}$, pressure 1 bar absolute), $\theta_{N}$ is temperature under normal conditions (273 Kelvin), $P_{N}$ is pressure under normal conditions ( 1 bar absolute), $\theta$ is process temperature in Kelvin and $P$ is the process pressure in bar absolute [9].

As pulse radar operates in the time domain, huge computational power requirements associated with Fast Fourier transform (FFT), as observed in Frequency-Modulated Continuous Wave (FMCW) counterpart, can be avoided. Emitted microwave pulses are regularly repeated with a time delay to allow return echo to be received before next pulse is transmitted. This period between the pulses is defined as inverse of pulse repetition frequency. Selected sensor configuration is shown in Figure 1(c). To achieve a highly focused beam and smaller process connection size, basic sensor working on $26 \mathrm{GHz}$ (K-band) frequency with a pulse repetition frequency of $3.6 \mathrm{MHz}$ was selected.

Isolation of sensor electronics from high process temperature was achieved by using a temperature isolator section of length $260 \mathrm{~mm}$. Antenna impedance cone of ceramic with graphite seal was selected for optimum transmission of microwaves and to provide appropriate sealing against high temperature application.

\subsection{Bulk Temperature Measurement and Temperature Profiling}

To measure bulk temperature of liquid $\mathrm{Pb}-\mathrm{Li}$ and to study feasibility of $\mathrm{Pb}-\mathrm{Li}$ level estimation using vertical bulk temperature profiling, a compact Temperature Level Probe (TeLePro) assembly was developed using a K-type multilevel thermocouple as shown in Figure 1(d). TeLePro provides a compact rigid probe for vertical temperature profiling of bulk medium which is adaptable for tanks with small diameter and tanks with internal installations. For this study, 21 temperature measuring junctions, separated by $20 \mathrm{~mm}$ each, were accommodated in a common outer sheath of length $400 \mathrm{~mm}$ and outer diameter of $6 \mathrm{~mm}$. Junction-1 corresponds to tip of sensor probe. A properly sized Inconel-600 thermowell protects the sensor probe from corrosion, process pressure and bending stresses. Level estimation using TeLePro is based on differential temperature measurement technique and can be further adapted to enhance the response and resolution by proper thermowell sizing and location of sensing points, respectively. Sensor customization might be limited by manufacturing feasibility and detectable temperature gradients.

\section{Experimental Details}

Two calibration test facilities were in-house designed for experimental validation of selected pressure, level and temperature sensors. These test facilities were designed to provide required test environments as well as to address installation constraints of selected sensors.

\subsection{Test Facility-1: Design Description and Test Procedure}

Test facility-1 was designed for calibration and performance validation of se- 
lected radar level sensor and silicone oil filled pressure sensor for liquid metal application. Major components of test facility-1 include main tank, top nozzle, side-section, drain tank and connecting pipeline with an isolation valve. Material of construction for these components is SS-316L. Schematic diagram of test facility-1 is shown in Figure 2(a) and an image of process sensors installed on the main tank is shown in Figure 2(b). Table 1 lists major process test parameters.

Main tank height $(600 \mathrm{~mm})$ was derived from the height of inventory tank planned for LLCS, while internal diameter of main tank $(492 \mathrm{~mm})$ and height of connection nozzle were primarily governed by installation constraints of level sensor to avoid false echoes. To achieve sufficient level variations in the large volume of main tank, liquid $\mathrm{Pb}$ was selected as an economical surrogate process medium. Drain tank, with an internal diameter of $202.7 \mathrm{~mm}$ and height of 630 $\mathrm{mm}$, allows for variation of liquid $\mathrm{Pb}$ level using cover gas pressurization technique. To the best of our knowledge, this is the first time a non-contact pulse radar level measurement technique is applied for measurement of liquid lead level. Side-section, with an adequate inclination for gravity assisted liquid metal draining, is provided for installation of silicone oil filled pressure sensor. Controlled shifting of liquid $\mathrm{Pb}$ between the tanks was achieved through manual operation of isolation valve. A secondary reference for liquid metal level estimation was established using conductivity level switches for discrete level measurements (Figure 3). Porcelain insulator of spark plug provided electrical isolation of electrode from tank structure.

For validation on liquid $\mathrm{Pb}$ application, radar level sensor was configured to measure level $(H)$ in the range of $0-694 \mathrm{~mm}$ as per the height of main tank with

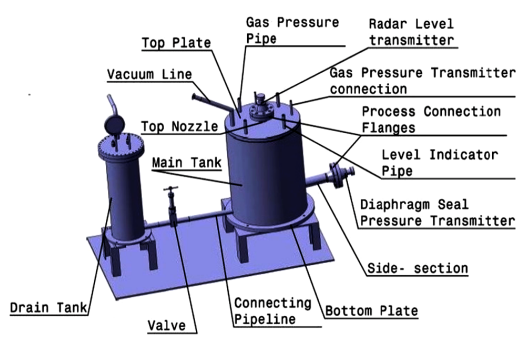

(a)

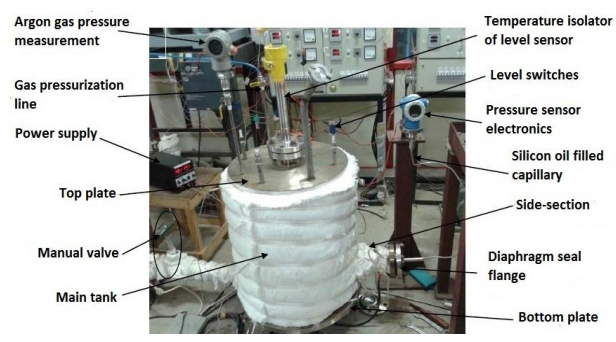

(b)

Figure 2. (a) Schematic diagram for test facility-1; (b) Image of test facility-1 main tank. Level sensor and pressure sensor are installed on the top nozzle and side-section, respectively.

Table 1. Process parameters for test facility-1.

\begin{tabular}{cc}
\hline Parameter & Value \\
\hline Process Medium & Liquid $\mathrm{Pb}$ \\
Operating Temperature & $380^{\circ} \mathrm{C}-400^{\circ} \mathrm{C}$ \\
Operating Pressure & Upto $1 \mathrm{MPa}$ (gauge) \\
Density of $\mathrm{Pb}$ at $400^{\circ} \mathrm{C}$ & $10,584 \mathrm{~kg} / \mathrm{m}^{3}[10]$ \\
Melting point of $\mathrm{Pb}$ & $327.4^{\circ} \mathrm{C}[10]$ \\
\hline
\end{tabular}




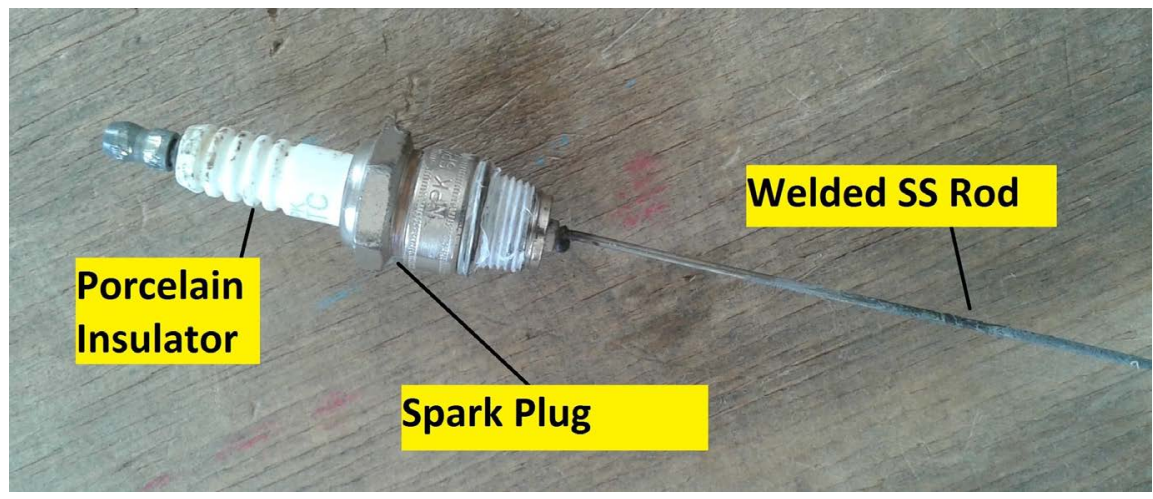

Figure 3. Conductivity level switch construction.

top nozzle. A known inventory (405 kg) in the form of solid $\mathrm{Pb}$ ingots was melted in main tank in an inert environment by applying positive pressure of argon cover gas. A reference calibrated gauge pressure sensor mounted on main tank measured cover gas pressure $\left(P_{g}\right)$. To perform first point calibration check, level of liquid $\mathrm{Pb}$ in main tank was analytically estimated using mass of $\mathrm{Pb}$ taken, density of $\mathrm{Pb}$ at operating temperature and dimensions of test facility- 1 and compared with output of radar level sensor. For second point calibration check, liquid $\mathrm{Pb}$ was transferred to drain tank upto a fixed level, estimated using a fixed conductivity level switch. The level of liquid $\mathrm{Pb}$ remaining in main tank was calculated and compared with the output of radar level sensor. All calculations take into account thermal expansion of both tanks along height to correct for zero level offset of radar level sensor and to correctly estimate liquid $\mathrm{Pb}$ level at operating temperature. Estimated radial expansions are negligible due to welded top and bottom plates.

After calibration checks, liquid $\mathrm{Pb}$ inventory was transferred to main tank for long duration test. Test was performed for over 700 hour continuously with cover gas pressure upto $1 \mathrm{MPa}(\mathrm{g})$ and readings were taken at regular intervals to validate the performance of radar level sensor.

For pressure sensor testing, diaphragm seal was installed on the side-section port. Zero adjustment for the elevation of sensor electronics mounting position was carried out. Sensor diaphragm seal was located $145 \mathrm{~mm}$ above the bottom of main tank, hence only a part of liquid $\mathrm{Pb}$ column $\left(H_{\text {effective }}\right)$ generated pressure $\left(P_{\text {effective }}\right)$ on the pressure sensor diaphragm.

$$
\begin{gathered}
H_{\text {effective }}=H-145 \mathrm{~mm} \\
P_{\text {effective }}=H_{\text {effective }} \cdot \rho_{\text {medium }} \cdot g
\end{gathered}
$$

where, $\rho_{\text {medium }}$ is density of $\mathrm{Pb}$ at operating temperature and $g$ is acceleration due to gravity. Total pressure $(P)$ exerted on diaphragm seal is summation of pressure applied through cover gas $\left(P_{g}\right)$ and pressure head due to effective liquid $\mathrm{Pb}$ column $\left(P_{\text {effective }}\right)$.

$$
P=P_{\text {effective }}+P_{g}
$$

From Equation (4), we can see that by varying cover gas pressure alone, one 
can vary total pressure applied to pressure sensor under test, while simultaneously ensuring diaphragm seal in direct contact with liquid $\mathrm{Pb}$. Calculated total pressure $P$ was compared with pressure sensor output to estimate the error. Testing was continuously done for over 310 hour and readings were taken at regular intervals.

\subsection{Test Facility-2: Design Description and Test Procedure}

Test facility-2 was fabricated for experimental validation of pressure sensors and TeLePro assembly in high-temperature, high-pressure liquid $\mathrm{Pb}$-Li environment. This study was intended to test compatibility of sensors with liquid $\mathrm{Pb}-\mathrm{Li}$, to understand deteriorating effects of corrosion on measurement reliabilities and to check feasibility of liquid $\mathrm{Pb}-\mathrm{Li}$ level estimation using TeLePro. Tank-A, with an internal diameter of $77.9 \mathrm{~mm}$ and height of $412 \mathrm{~mm}$, was used to test both types of pressure sensors simultaneously on two side-sections while tank-B, with an internal diameter of $52.5 \mathrm{~mm}$ and height of $528 \mathrm{~mm}$, was used for testing of TeLePro assembly. Tubes were introduced between end of each side-section and tank-A top volume to remove trapped gas volume during charging of $\mathrm{Pb}-\mathrm{Li}$ as well as to help ensure proper drainage of liquid $\mathrm{Pb}$-Li from side-sections using active gas pressurization. Testing of pressure sensors and TeLePro assembly were performed sequentially as shown in Figure 4(a) and Figure 4(b). Process test parameters are listed in Table 2.

For pressure sensors calibration and testing, temperatures of both the sidesections were maintained between $380^{\circ} \mathrm{C}-400^{\circ} \mathrm{C}$. Effective $\mathrm{Pb}-\mathrm{Li}$ head for each pressure sensor was estimated through an average of repeated measurements done using movable conductivity type level switch. For silicone oil filled pressure sensor, $H 1_{\text {effective }}$ was $66 \mathrm{~mm}$ and for $\mathrm{NaK}$ filled pressure sensor, $H 2_{\text {effective }}$ was 75 $\mathrm{mm}$. Total exerted pressures on the pressure sensors were estimated using Equations (3) and (4) where density of $\mathrm{Pb}-\mathrm{Li}$ at $400^{\circ} \mathrm{C}$ was estimated using empirical relation [11]. Calculated total exerted pressures were compared with sensor outputs to estimate the error. Two calibration cycles, each from 0 to $1 \mathrm{MPa}(\mathrm{g})$ and vice-versa, were performed at start and end of continuous 1000 hour long duration test. Cover gas pressure was maintained in tank-A and readings were taken at regular intervals.

The customized TeLePro assembly is shown in Figure 5. TeLePro was subjected to severe thermal aging in corrosive liquid $\mathrm{Pb}-\mathrm{Li}$ with cover gas pressure upto $1 \mathrm{MPa}(\mathrm{g})$. For first 500 hour, heater of tank-B was controlled using surface temperature measurement and for next 500 hour, junction-1 of TeLePro was used for heater control using bulk $\mathrm{Pb}$ - $\mathrm{Li}$ temperature measurement. Maximum bulk temperature upto $520^{\circ} \mathrm{C}$ was attained for extended durations. After completion of continuous 1000 hour long duration test, TeLePro development campaign for liquid $\mathrm{Pb}$-Li level estimation was carried out. In principle, a reliable level sensing technique should be unaffected by process temperature and pressure conditions. To validate the assembly, steady state temperature profiles were 
obtained for two cases; Case-I: different cover gas pressures at a constant temperature Control Set-Point (CSP) and Case-II: different temperature CSPs at a

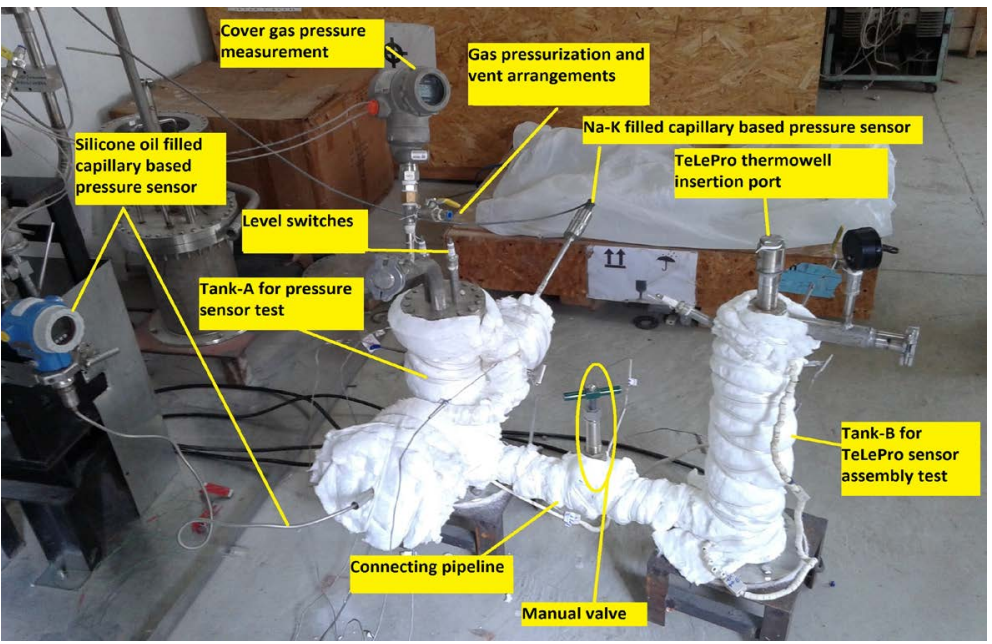

(a)

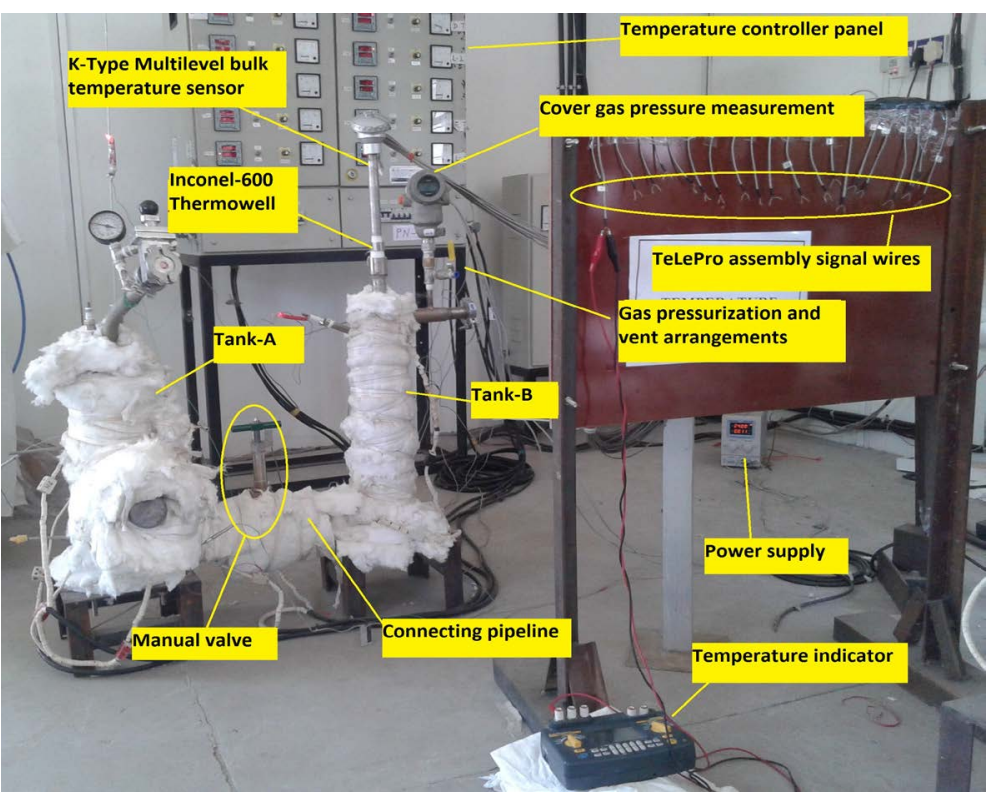

(b)

Figure 4. (a) Pressure sensors testing phase in test facility-2; (b) TeLePro assembly testing phase in test facility- 2 .

Table 2. Process parameters for test facility- 2 .

\begin{tabular}{cc}
\hline Parameter & Value \\
\hline Process Medium & Liquid $\mathrm{Pb}-16 \mathrm{Li}$ \\
Operating Temperature & $250^{\circ} \mathrm{C}-530^{\circ} \mathrm{C}$ \\
Operating Pressure & Upto $1.05 \mathrm{MPa}$ (gauge) \\
Density of Pb-16Li at $400^{\circ} \mathrm{C}$ & $9318 \mathrm{~kg} / \mathrm{m}^{3}[11]$ \\
Melting point of Pb-16Li & $235^{\circ} \mathrm{C}[11]$ \\
\hline
\end{tabular}




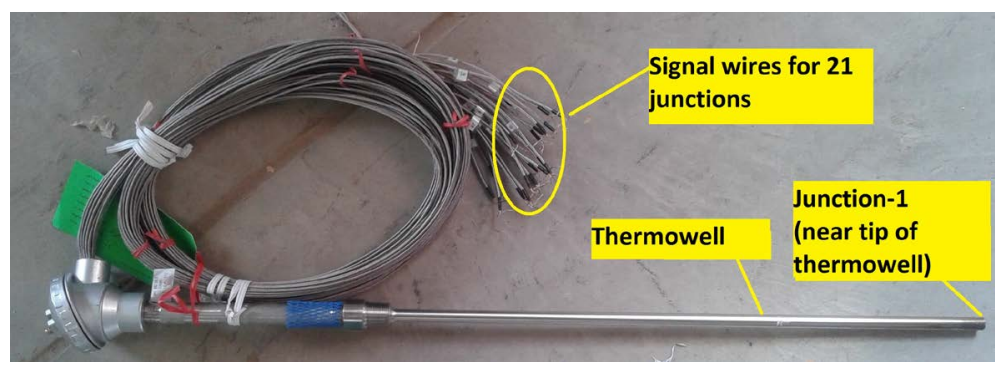

Figure 5. TeLePro assembly before exposure to liquid metal.

constant cover gas pressure. Sensor assembly was exposed to liquid $\mathrm{Pb}-\mathrm{Li}$ continuously for over 1240 hour. After completion of testing, TeLePro assembly was removed in hot condition from the facility.

\section{Results and Discussions}

For first point analytical calibration of radar level sensor, estimated liquid $\mathrm{Pb}$ level in main tank was $198.42 \mathrm{~mm}$ and observed sensor reading was $204.97 \mathrm{~mm}$. Thus a deviation of $+6.55 \mathrm{~mm}$ was observed. For second point calibration, analytically estimated level in main tank was $104.28 \mathrm{~mm}$ and observed sensor reading was $116.66 \mathrm{~mm}$. Hence, a deviation of $+12.38 \mathrm{~mm}$ was observed. It should be noted that before employing radar level sensor on liquid $\mathrm{Pb}$ application, calibration checks were performed under ambient conditions using 1) a metallic reflector plate and 2) water as process fluid.

During these tests, observed error was within $[+1 \mathrm{~mm},+5 \mathrm{~mm}]$, which is close to as suggested by datasheet. A few echo curves were also obtained during liquid $\mathrm{Pb}$ level measurements. Figure 6(a) shows an observed echo pattern from liquid $\mathrm{Pb}$ surface along with preset false signal suppression curve. This echo suggests an average distance of liquid $\mathrm{Pb}$ surface at $495 \mathrm{~mm}$ from reference plane of top nozzle, which corresponds to liquid $\mathrm{Pb}$ level of $\sim 203 \mathrm{~mm}$ from main tank bottom. During design, all sources of false echoes were taken care of, hence preset false signal suppression curve was removed to obtain clean liquid metal echo of interest as shown in Figure 6(b), which suggests liquid $\mathrm{Pb}$ level of $\sim 199 \mathrm{~mm}$. In both the cases, observed echo amplitudes $(>60 \mathrm{~dB})$ affirm strong reflected echoes.

Figure 7 presents long duration test data for liquid $\mathrm{Pb}$ level measurement. The obtained data suggests absence of smooth surface which may be attributed to level readings averaged over a surface area containing floating oxide layers with multifaceted surface topography on top of the melt. Estimated deviation in level measurement of liquid $\mathrm{Pb}$ was within $[-3.36 \mathrm{~mm},+13.64 \mathrm{~mm}]$ for over 1000 hour duration. Sources of error include assumption of constant bulk density of liquid $\mathrm{Pb}$ for analytical estimation of true level, error in level detection using conductivity switch and measurement accuracy of sensor itself. However, accuracy of radar level sensor re-confirmed on water application was better than $3 \mathrm{~mm}$, while errors due to other sources were estimated very small. Hence, 


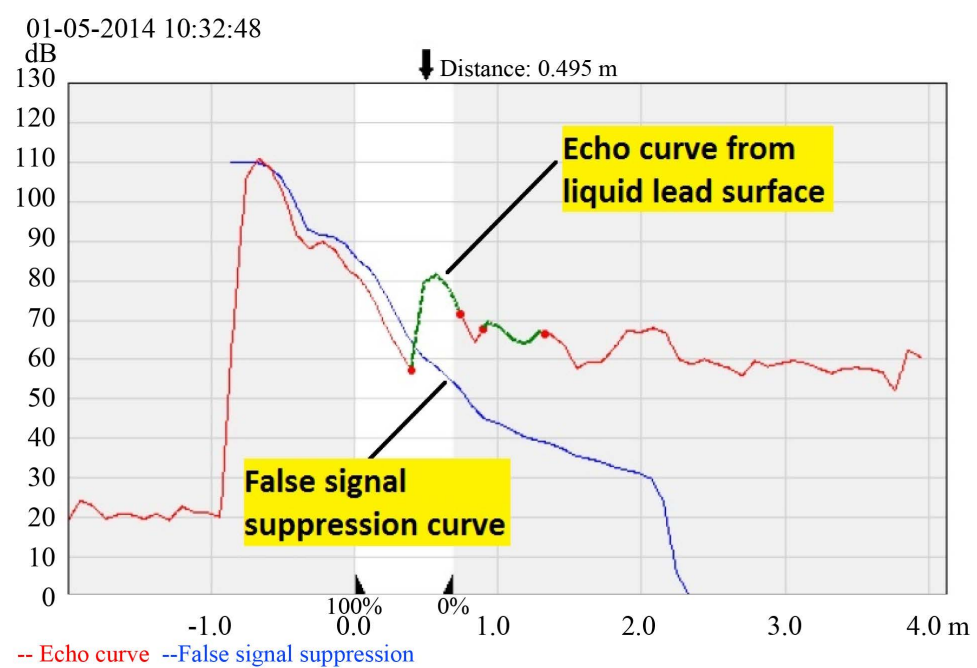

(a)

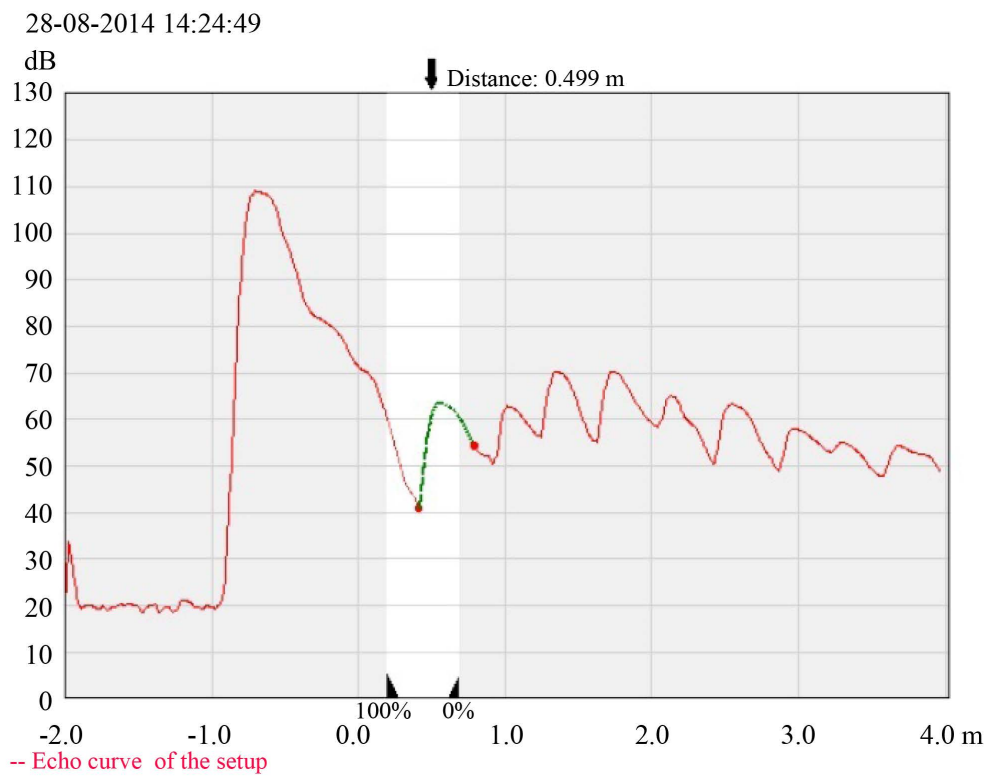

(b)

Figure 6. (a) Echo observed at $495 \mathrm{~mm}$ (from radar level sensor reference plane) during liquid $\mathrm{Pb}$ level measurements (preset false signal suppression curve is also shown); (b) Echo observed at $499 \mathrm{~mm}$ (from radar level sensor reference plane) during liquid $\mathrm{Pb}$ level measurements after removing false signal suppression curve.

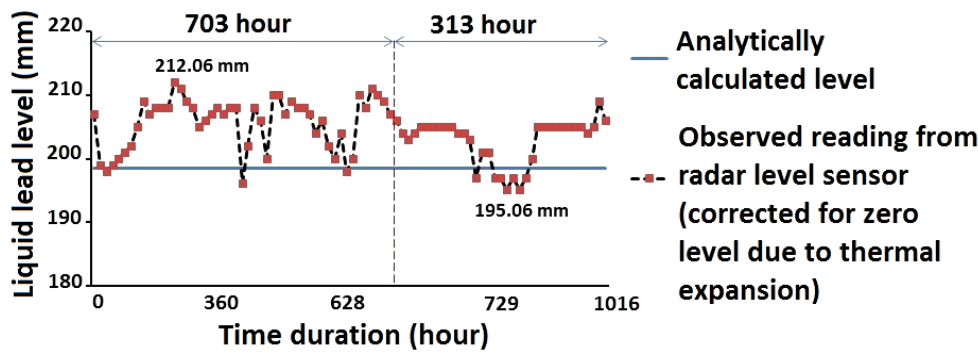

Figure 7. Long duration test data from non-contact radar level sensor suggests absence of smooth liquid $\mathrm{Pb}$ surface. 
observed deviation seems largely due to irregular surface profile of oxide layers. Presence of such irregular oxide layers was later visually confirmed in similar applications. In future experiments, a separate melt tank is planned to melt and transfer clean liquid metal in the main inventory tank. As the selected radar level sensor is non-contact configuration, its calibration and rigorous validation for liquid $\mathrm{Pb}$ also corroborates its suitability for other liquid metals/metallic alloys including $\mathrm{Pb}$-Li.

Estimated error in pressure measurement of liquid $\mathrm{Pb}$ using silicon oil filled pressure sensor was within $0.3 \%$ of calibrated span for over 310 hour test duration. Figure 8 presents long duration test data for pressure measurement of liquid $\mathrm{Pb}$.

Figure 9(a) and Figure 9(b) show test results obtained and condition of diaphragm seals after long term exposure to $\mathrm{Pb}-\mathrm{Li}$ at high temperature and pressure. NaK filled pressure sensor showed good agreement with total applied pressure for complete test duration including calibration cycles and the estimated error lies within $1.1 \%$ of calibrated span for over 1000 hour test duration.

Silicone oil filled pressure sensor showed good agreement with total applied pressure during initial two calibration cycles and during long duration test. Estimated error lies within $0.9 \%$ of calibrated span for this test duration. But at start of third calibration cycle, silicone oil filled pressure sensor did not follow total applied pressure and displayed a gauge pressure reading between $0.4 \mathrm{MPa}$ $0.46 \mathrm{MPa}$ for all applied pressures less than 0.4 MPa. Visual inspection confirmed deposition of $\mathrm{Pb}-\mathrm{Li}$ (with lustrous silvery characteristics) over seal diaphragm of $\mathrm{NaK}$ filled pressure sensor while seal diaphragm of silicone oil filled pressure sensor was partially covered with lustrous $\mathrm{Pb}-\mathrm{Li}$ and partially with a greyish-blackish layer. X-Ray Diffraction (XRD) analysis of samples taken from corresponding side-sections indicated presence of $\mathrm{PbO}$ and $\mathrm{Li}_{2} \mathrm{O}$. This oxide formation can occur due to various factors including presence of free oxygen, $\mathrm{Li}_{2} \mathrm{O}$ and $\mathrm{PbO}$ in the used $\mathrm{Pb}-\mathrm{Li}$ ingots and surface oxidation of ingots during long term storage. The abrupt behavior observed for silicone oil filled pressure sensor could be due to damage/distortion of seal diaphragm, formation and

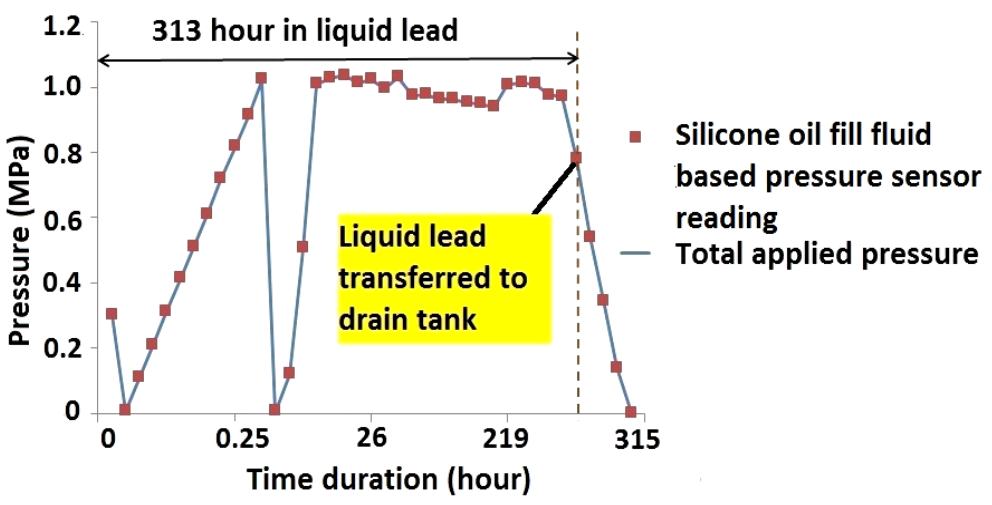

Figure 8. Calibration cycle and long duration test data for silicone oil fill fluid based pressure sensor on liquid $\mathrm{Pb}$. 

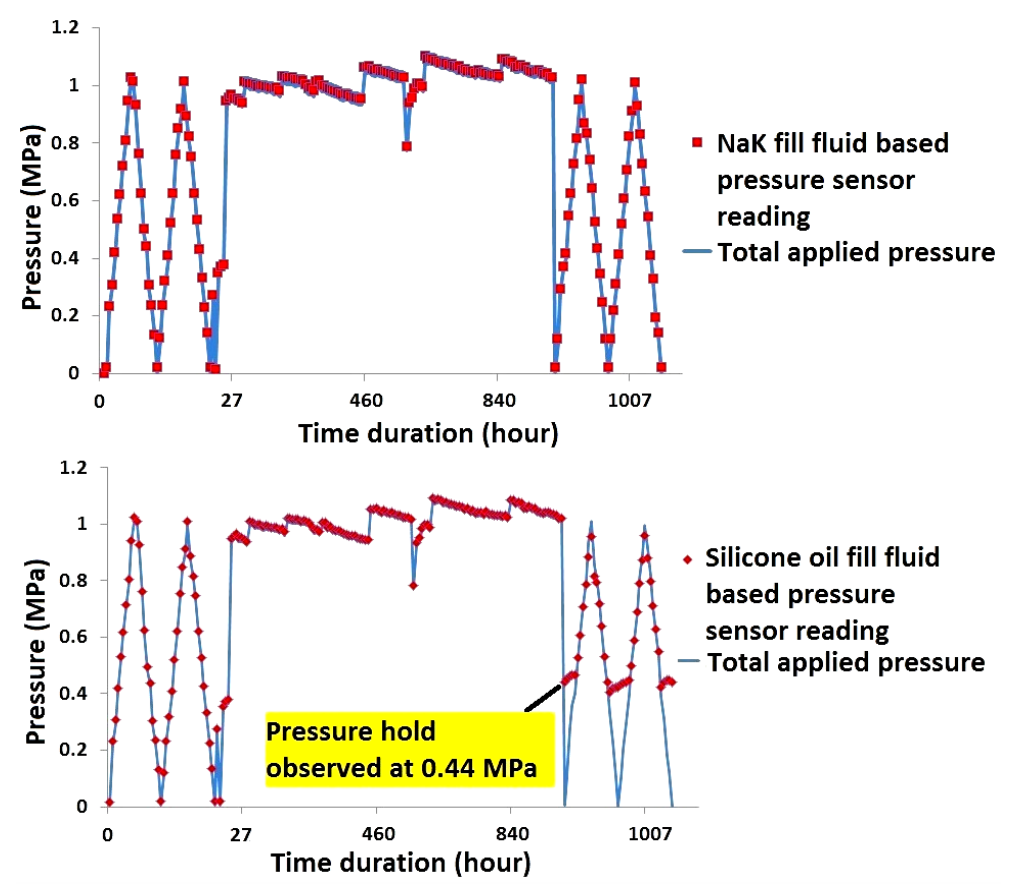

(a)
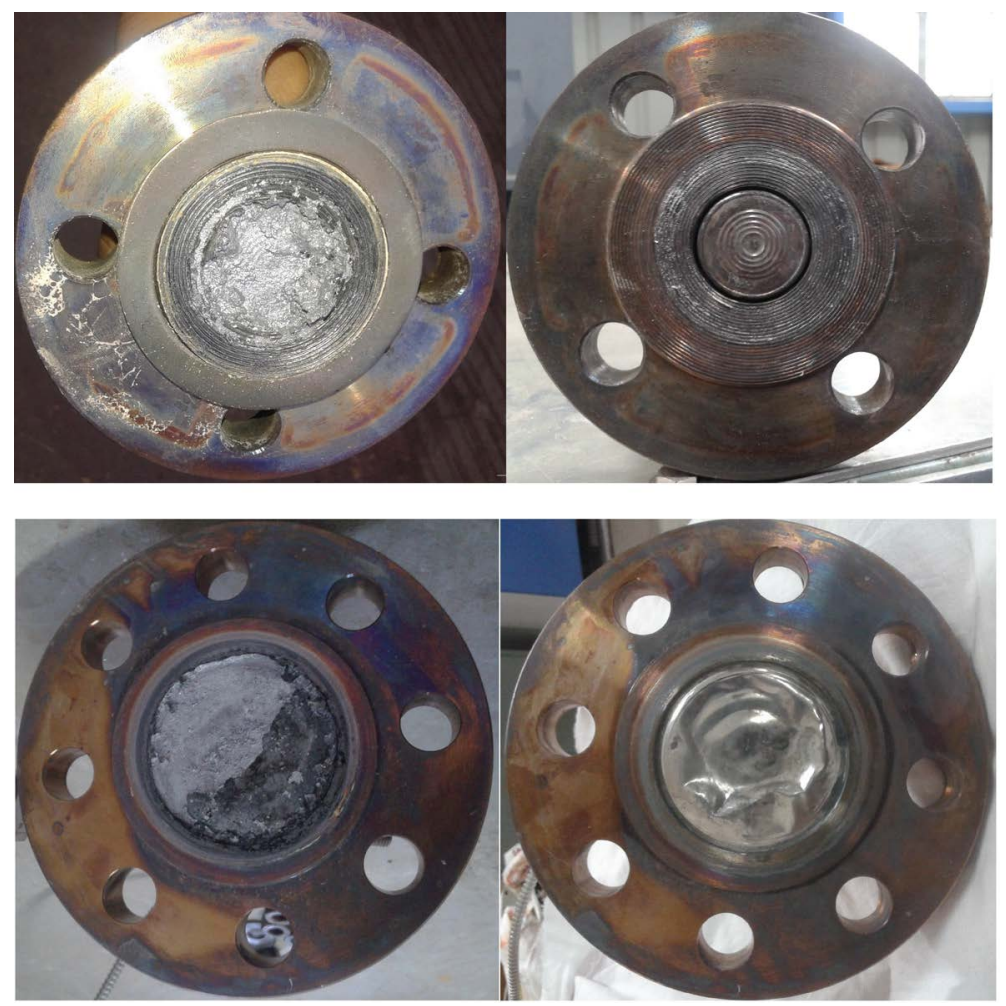

(b)

Figure 9. (a) Long duration test data for $\mathrm{NaK}$ fill fluid based and silicone oil fill fluid based pressure sensors on $\mathrm{Pb}$ - $\mathrm{Li}$ (gradual changes in applied pressure represent decrease of pressure with time). M-shaped cycles at the start and end of long duration test represent calibration cycles from 0 to $1 \mathrm{MPa}$ (g) and vice-versa; (b) Condition of wetted diaphragm seals for $\mathrm{NaK}$ fill fluid based and silicone oil fill fluid based pressure sensors after over 1000 hour continuous exposure to liquid $\mathrm{Pb}-\mathrm{Li}$ and after chemical cleaning. 
deposition of oxides on the seal diaphragm, thermal expansion of silicone oil inside the capillary or could be a combination of one or more of the above factors. Further diagnosis was carried out by providing heat to diaphragm seal at ambient pressure, where pressure sensor displayed a substantial increase (upto 0.38 MPa gauge) in measured value with rise in temperature at ambient pressure. This behavior suggests thermal expansion of silicone oil leading to pressurization of measuring cell membrane.

For further inspection, diaphragm seals were chemically cleaned by immersing in an equal volume mixture of acetic acid, hydrogen peroxide and ethyl alcohol followed by cleaning with water [12]. The diaphragm seal of silicone oil filled pressure sensor displayed signature of distortion. In a similar trial with an identical silicone oil filled pressure sensor, after 160 hour of continuous exposure to liquid $\mathrm{Pb}$ followed by continuous 210 hour in liquid $\mathrm{Pb}-\mathrm{Li}$, a similar pressure reading hold was observed between $0.42 \mathrm{MPa}-0.44 \mathrm{MPa}$ for all applied pressures less than 0.4 MPa, suggesting prominent thermal expansion of silicone oil inside the capillary at temperatures near $400^{\circ} \mathrm{C}$. These observations indicate ineffectiveness of silicone oil fill fluid for long duration operations in high temperature liquid $\mathrm{Pb} / \mathrm{Pb}-\mathrm{Li}$ applications relevant to LLCS.

For TeLePro development as a liquid $\mathrm{Pb}$-Li level estimation technique, tank-B surface heater was controlled using a single point bulk $\mathrm{Pb}-\mathrm{Li}$ temperature measurement with junction-1 of TeLePro. Higher thermal conductivity of $\mathrm{Pb}-\mathrm{Li}$ would allow more heat transfer from constantly heated tank walls to the TeLePro through bulk $\mathrm{Pb}$ - $\mathrm{Li}$. While, above $\mathrm{Pb}$-Li melt, due to presence of oxides layer and cover gas region with relatively lower thermal conductivity, transferred heat towards TeLePro would be less, leading to a reduction in measured temperature by TeLePro junctions. Hence a sharp decrease in temperature was expected in oxides and cover gas region leading to interface detection. Steady state temperature profiles obtained are shown in Figure 10(a) and Figure 10(b). Observed temperature profiles depict thermal stratification within the thermally insulated tank- $\mathrm{B}$, in which static $\mathrm{Pb}-\mathrm{Li}$ column is arranged into layers of different densities. Hence, an increasing temperature profile is observed within bulk $\mathrm{Pb}-\mathrm{Li}$ starting from junction- 1 upto the $\mathrm{Pb}-\mathrm{Li}$ surface.

As observed in Case-I, temperature for the region near Pb-Li top surface decreased with an increase in cover gas pressure while bulk $\mathrm{Pb}-\mathrm{Li}$ temperature measured by lower junctions remained more or less the same. Observed profiles could be explained by the fact that the number of gas molecules increased with increase in gas pressure, aiding in more heat removal from $\mathrm{Pb}$-Li surface region.

In contrast, during Case-II, temperature profiles show an overall shift upwards with an increase in temperature CSP due to an effective bulk temperature rise for $\mathrm{Pb}-\mathrm{Li}$, oxides region and cover gas. Obtained profiles from both the cases show that temperature increased continuously from TeLePro junction-1 to junction- 15 , remained nearly constant (within $3^{\circ} \mathrm{C}$ ) from junction- 15 to junction- 18 and thereafter decreased continuously. For all observed cases, obtained temperature drops were $\Delta_{18-19}=7^{\circ} \mathrm{C}$ to $12^{\circ} \mathrm{C}, \Delta_{19-20}=17^{\circ} \mathrm{C}$ to $25^{\circ} \mathrm{C}$ and $\Delta_{20-21}=20^{\circ} \mathrm{C}$ to 
$29^{\circ} \mathrm{C}$ where $\Delta_{\mathrm{a}-\mathrm{b}}$ denotes temperature drop from junction a to junction b. Observed pattern and magnitude of temperature drop indicated liquid $\mathrm{Pb}-\mathrm{Li}$ surface below junction-19, but very close to junction-19. Junction-19 lies at 366.4 $\mathrm{mm}$ from the thermowell tip as per TeLePro design. TeLePro assembly was removed after over 1240 hour of continuous exposure and a clear demarcation was observed on the thermowell with deposited greyish layers starting at a distance of nearly $370 \mathrm{~mm}$ from thermowell tip. This visual observation further corroborates that liquid $\mathrm{Pb}$-Li level estimation can be done within reasonable accuracy limits using TeLePro. In the proposed sensor assembly, precise level estimation is governed by resolution, which is defined by the physical separation between successive temperature sensing junctions. Figure 11 shows observed deposited layers on TeLePro thermowell. XRD analysis indicated $\mathrm{PbO}$ and $\mathrm{Li}_{2} \mathrm{O}$ in the top deposited greyish layers while no evidence of nickel was present in the samples taken from different locations along the length of thermowell, suggesting negligible corrosion over this test period. Sensor probe was observed completely clean and protected from corrosion. The obtained preliminary results from

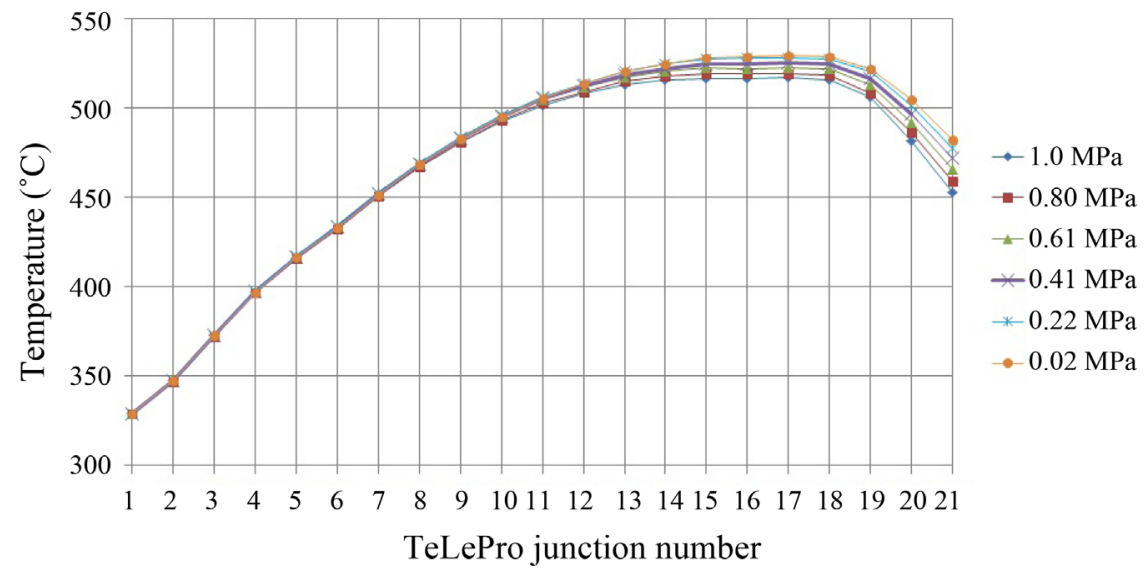

(a)

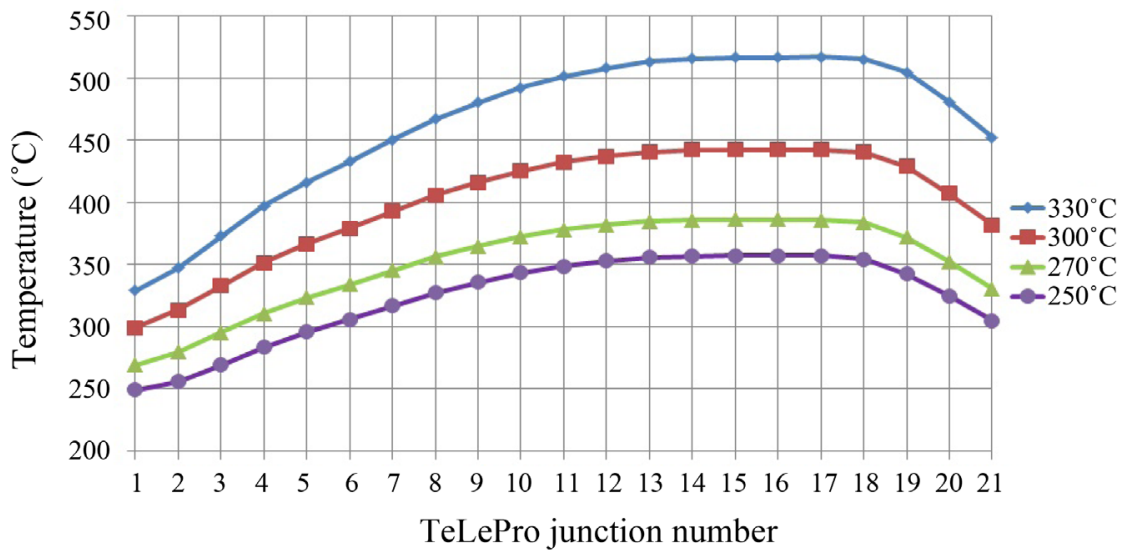

(b)

Figure 10. (a) Case-I: Temperature profiles at constant CSP of $330^{\circ} \mathrm{C}$ with different cover gas pressures; (b) Case-II: Temperature profiles at constant gas pressure of $1 \pm 0.01 \mathrm{MPa}$ (g) with different CSPs. 


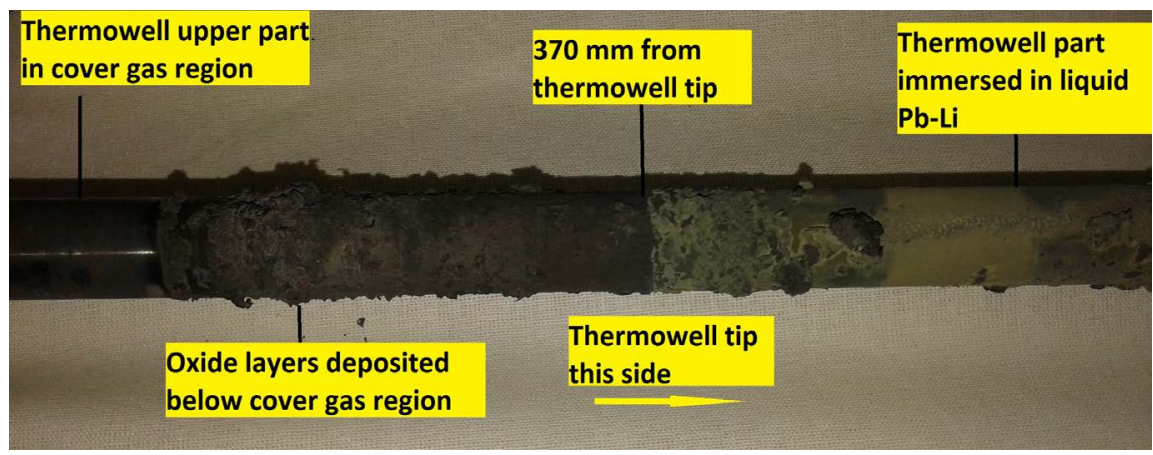

Figure 11. Observed deposition of greyish oxide layers starting at $370 \mathrm{~mm}$ (from the tip) on TeLePro thermowell.

TeLePro tests are encouraging. However, further tests are required for calibration of TeLePro against a reference level sensor to detect interface between bulk liquid $\mathrm{Pb}-\mathrm{Li}$ and cover gas/oxide layers on the top of $\mathrm{Pb}-\mathrm{Li}$.

\section{Conclusion}

Since instrumentation availability is limited for liquid metal applications, rigorous test methods were applied to develop and validate pressure, level and bulk temperature diagnostics for static liquid $\mathrm{Pb} / \mathrm{Pb}-\mathrm{Li}$ applications. Indigenous calibration test facilities were designed and fabricated at IPR to provide required test environments over long durations. A differential temperature measurement based interface detection technique using bulk temperature profiling was also proposed and extensively studied. During these preliminary studies, high reliability and availability was observed for $\mathrm{NaK}$ filled remote diaphragm seal type wetted pressure sensor, non-contact pulse radar level sensor and bulk temperature sensor. Performed studies suggest that silicone oil is not suitable as a high-temperature de-coupling fill fluid for long duration applications due to significant thermal expansion. As such, no impact of corrosion was observed on the measurement accuracies of wetted sensors during 1000 hour tests. Performed static tests represent scenarios similar to foreseen measurement applications in LLCS, because all these sensor configurations are planned to be installed in nearly static liquid Pb-Li conditions. Further design optimizations and compatibility of process sensors with environmental factors (like magnetic field, radiation etc.) still need to be addressed for a complete development relevant to applications in heavy liquid metal coolant systems for fusion test blankets. Obtained performance data from validated and optimized sensor configurations may be useful for RAMI and FMEA analysis at component level for ancillary plant systems related to LLCB TBM applications.

\section{Acknowledgements}

Authors would like to acknowledge the assistance rendered by A. Jaiswal (IPR) and K. Pandya (IPR) for the operation and maintenance of test facilities. In addition, authors deeply appreciate the support from C.S. Sasmal (IPR) for the 
metallographic work and analysis of samples from wetted sensors.

\section{References}

[1] Giancarli, L.M., et al. (2012) Overview of the ITER TBM Program. Fusion Engineering and Design, 87, 395-402. https://doi.org/10.1016/j.fusengdes.2011.11.005

[2] Indian TBM Design Team (2008) Design Description Document (DDD) for "Lead Lithium cooled Ceramic Breeder (LLCB) Blanket”. Version1.0, April 2008.

[3] Chaudhuri, P., et al. (2012) Status and Progress of Indian LLCB Test Blanket Systems for ITER. Fusion Engineering and Design, 87, 1009-1013.

https://doi.org/10.1016/j.fusengdes.2012.02.062

[4] Kitazawa, S.-I., Okayama, K., Neyatani, Y., Sagot, F. and van Houtte, D. (2014) RAMI Analysis of the ITER CIS. Fusion Engineering and Design, 89, 88-93. https://doi.org/10.1016/j.fusengdes.2013.12.016

[5] International Atomic Energy Agency (2012) Liquid Metal Coolants for Fast Reactors Cooled by Sodium, Lead and Lead-Bismuth Eutectic, IAEA NUCLEAR ENERGY SERIES No. NP-T-1.6, STI/PUB/1567.

[6] Mas de les Valls, E., Sedano, L.A., Batet, L., Ricapito, I., Aiello, A., Gastaldi, O. and Gabriel, F. (2008) Lead-Lithium Eutectic Material Database for Nuclear Fusion Technology. Journal of Nuclear Materials, 376, 353-357.

https://doi.org/10.1016/j.jnucmat.2008.02.016

[7] Wetzel, T. (2015) Chapter 11: Instrumentation, Handbook on Lead-Bismuth Eutectic Alloy and Lead Properties, Materials Compatibility, Thermal-Hydraulics and Technologies. OECD, NEA. No. 7268, Nuclear Science.

[8] Smolentsev, S., Li, F.-C., Morley, N., Ueki, Y., Abdou, M. and Sketchley, T. (2013) Construction and Initial Operation of MHD PbLi Facility at UCLA. Fusion Engineering and Design, 88, 317-326. https://doi.org/10.1016/j.fusengdes.2013.03.018

[9] Devine, P. (2000) Radar Level Measurement-The User's Guide. VEGA Controls.

[10] Sobolev, V. (2011) Database of Thermophysical Properties of Liquid Metal Coolants for GEN-IV. 2nd Edition, Scientific Report of the Belgian Nuclear Research Centre, Belgium, $173 \mathrm{p}$.

[11] Schulz, B. (1991) Thermophysical Properties of the $\mathrm{Li}(17) \mathrm{Pb}(83)$ Alloy. Fusion Engineering and Design, 14, 199-205. https://doi.org/10.1016/0920-3796(91)90002-8

[12] Glasbrenner, H., Konys, J., Rohrig, H.D., Stein-Fechner, K. and Voss, Z. (2000) Corrosion of Ferritic-Martensitic Steels in the Eutectic Pb-17Li. Journal of Nuclear Materials, 283-287, 1332-1335. https://doi.org/10.1016/S0022-3115(00)00322-6 